See Article page XXX.

\section{Commentary: Postoperative atrial fibrillation after cardiac surgery: Canary in a coal mine?}

\author{
Sari D. Holmes, $\mathrm{PhD},{ }^{\mathrm{a}}$ and Niv $\mathrm{Ad}, \mathrm{MD}^{\mathrm{a}, \mathrm{b}}$
}

In their study, Bianco and colleagues ${ }^{1}$ found an association between early postoperative atrial fibrillation (POAF) after cardiac surgery and inferior long-term outcomes. Based on their large-scale study, including a robust propensity-matched analysis, they report that patients with early POAF had reduced survival and greater risk for readmissions during up to 5 years of follow-up.

There are several relevant variables to consider when evaluating these findings. First, the accuracy of preoperative AF status may conflate the findings for POAF if patients were included in the study with undetected preoperative AF history, which in turn significantly influences longterm outcomes. Evidence from our work and the Left Atrial Appendage Occlusion Study III trial found reduced survival and greater stroke risk, respectively, for patients with preoperative AF that was untreated during cardiac surgery. ${ }^{2-4}$ Parsing out the influence of true new-onset POAF is a common challenge for observational studies that rely on prior history of $\mathrm{AF}$ for exclusion criteria, without evaluating $\mathrm{AF}$ at the time of surgery.

Second, not all POAF is the same. Bianco and colleagues ${ }^{1}$ found that POAF was related to higher incidence of other perioperative morbidities, including reoperation, blood transfusion, sepsis, prolonged ventilation, pneumonia, perioperative intra-aortic balloon pump, renal failure, and new-onset dialysis. For patients who experienced complications, POAF is part of a complex postoperative

\footnotetext{
From the a Division of Cardiac Surgery, University of Maryland School of Medicine, Baltimore, Md; and ${ }^{\mathrm{b}}$ Adventist White Oak Medical Center, Silver Spring, Md.

Disclosures: Dr Ad has relationships with Medtronic, AtriCure, LivaNova, and Left Atrial Appendage Occlusion, LLC. Dr Holmes reported no conflicts of interest.

The Journal policy requires editors and reviewers to disclose conflicts of interest and to decline handling or reviewing manuscripts for which they may have a conflict of interest. The editors and reviewers of this article have no conflicts of interest.

Received for publication Nov 6, 2021; revisions received Nov 6, 2021; accepted for publication Nov 8, 2021.

Address for reprints: Sari D. Holmes, PhD, Division of Cardiac Surgery, University of Maryland School of Medicine, 110 S Paca St, 7th Floor, Baltimore, MD 21201 (E-mail: Sari.Holmes@som.umaryland.edu).

J Thorac Cardiovasc Surg 2021; $\mathbf{\square}: 1-2$

$0022-5223 / \$ 36.00$

Copyright (C) 2021 by The American Association for Thoracic Surgery

https://doi.org/10.1016/j.jtcvs.2021.11.013
}

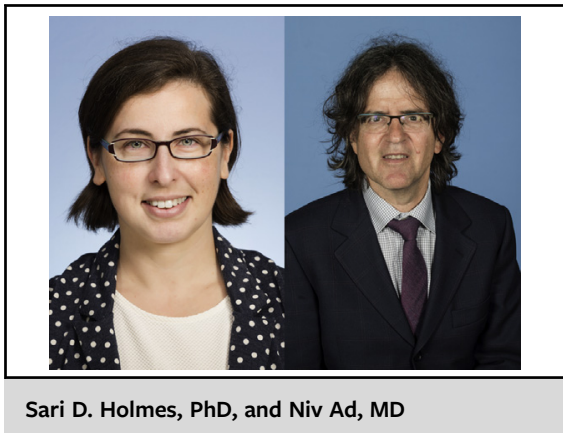

CENTRAL MESSAGE

Relevant factors to consider regarding these results include the role of undetected preoperative $A F$, misattributing risk to all POAF experiences, and the influence of incomplete follow-up.

course that could include low cardiac output, inotropes, and excessive bleeding. However, there are also patients who have no complications and may simply experience a short duration of POAF while in the step-down unit. It is unclear whether or not these 2 experiences with POAF after cardiac surgery will result in the same short- and long-term outcomes. Although this study adjusted for perioperative complications in the unmatched analyses of long-term outcomes, it is unclear whether or not group membership for patients with POAF represents a uniform experience. The findings for worse long-term outcomes with POAF may be driven by the experience of a complicated postoperative course and by patients who had additional recurrence of $\mathrm{AF}$ into a more persistent status. As the authors point out, POAF may serve as a marker for this process rather than a direct causal factor. Focusing on the risk from any occurrence of AF during the postoperative course may be imprecise and misdirect from the more influential factors.

Lastly, the methodology for assessing long-term outcomes can provide challenges from missing data and accuracy of assuming no events occurred based on absence of information. One study found that incomplete follow-up led to underestimation of mortality. ${ }^{5}$ There is no evidence that the bias associated with incomplete follow-up and missing data is disproportionately applied to the 2 groups 
in this study. However, knowing patients with POAF were more likely to have other perioperative complications, it is possible that more thorough, routine follow-up was conducted for these patients, allowing for more follow-up events to be uncovered.

This study emphasizes the potential long-lasting influence of POAF following cardiac surgery. Bianco and colleagues ${ }^{1}$ highlight the importance of closer monitoring for patients who experienced new-onset POAF and additional AF management when necessary to prevent further complications.

\section{References}

1. Bianco V, Kilic A, Yousef S, et al. The long-term impact of postoperative atrial fibrillation after cardiac surgery. J Thorac Cardiovasc Surg. 2022. XXX:XXX.

2. Ad N, Holmes SD, Massimiano PS, Pritchard G, Stone LE, Henry L. The effect of the Cox-maze procedure for atrial fibrillation concomitant to mitral and tricuspid valve surgery. J Thorac Cardiovasc Surg. 2013;146:1426-35.

3. Whitlock RP, Belley-Cote EP, Paparella D, Healey JS, Brady K, Sharma M, et al. Left atrial appendage occlusion during cardiac surgery to prevent stroke. $N$ Engl J Med. 2021;384:2081-91.

4. Whitlock RP, Belley-Cote EP. Patients with atrial fibrillation undergoing cardiac surgery: no left atrial appendage shall remain untouched. Innovations. 2021;16:301-2.

5. von Allmen RS, Weiss S, Tevaearai HT, Kuemmerli C, Tinner C, Carrel TP, et al. Completeness of follow-up determines validity of study findings: results of a prospective repeated measures cohort study. PLoS One. 2015;10:e0140817. 